\title{
Microscopic Theory of Gilbert Damping for Transition Metal Systems
}

\author{
Akimasa Sakuma \\ Department of Applied Physics, Tohoku University, Aoba-ku, Sendai, 980-8579, Japan
}

\begin{abstract}
We provide an overview on the microscopic theory of Gilbert damping and a theoretical framework to calculate the damping constant. In general, the damping constant can be expressed using the spin susceptibility of electronic system using the molecular field Hamiltonian. For a uniform magnetic dynamics (Kittel mode), magnetic damping does not occur unless the magnetic scatterings or spin-orbit interactions exist. Based on these concepts, we have performed the first principles calculation for the Gilbert damping constants $\alpha$ of transition metal systems such as Fe-Ni and Fe-Pt using the tight-binding linear muffin-tin orbital (TB-LMTO) method with inclusion of spin-orbit interactions. Quantitatively, the calculated $\alpha$ 's are approximately half of the experimental values, whereas the variations in the Fermi level dependence of $\alpha$ are much larger than these discrepancies. As expected, we confirm that for $\left(\mathrm{Fe}_{-} \mathrm{Ni}\right)_{1-\mathrm{x}} \mathrm{Ptx}$ and $\mathrm{FePt}$ systems the $\mathrm{Pt}$ atoms enhance $\alpha$ due to their large spin-orbit coupling. For the disordered alloys, we find that $\alpha$ decreases with an increasing chemical degree of order in the wide range.
\end{abstract}

Key words: Landau-Lifshitz-Gilbert equation, Gilbert damping constant, spintronics, spin relaxation, first principles calculations, TB-LMTO, CPA

\section{Introduction}

The recent development of magnetic devices such as magnetic recording systems and magnetic random access memory (MRAM) requires the realization of high speed (action time of less than $10 \mathrm{ns)} \mathrm{and} \mathrm{low}$ consumption power. For example, current research has mainly been focusing on achieving low consumption power in MRAM by using the current induced magnetization reversal phenomenon instead of applying external magnetic fields. The main goal of research on the magnetization reversal phenomenon is to reduce the critical current density $(J \mathrm{c})$ to an order of $10^{5} \mathrm{~A} / \mathrm{cm}^{2}$, and much effort has been devoted to accomplish this task. In 1996, Slonczewski ${ }^{1)}$ reported that $J \mathrm{c}$ is proportional to the Gilbert damping coefficient $\alpha$, which appears in the Landau-Lifshitz-Gilbert (LLG) equation that is widely used for studying the dynamics of magnetization. Generally, $\alpha$ is believed to dominate the magnetization reversal time. Additionally, based on the general concept of damping, $\alpha$ is also considered to be related to the dephasing time of spin precessions, which is currently an important concern in the development of quantum computing systems. Thus, the ability to control the damping is becoming a key factor in the development, and accordingly the microscopic foundation of the dynamical behavior (particularly, magnetization reversal process) is needed in this field.

The LLG equation is given by

$$
\dot{\boldsymbol{M}}=-\gamma \boldsymbol{M} \times \boldsymbol{H}_{\text {eff }}+\alpha \frac{\boldsymbol{M}}{\boldsymbol{M}} \times \dot{\boldsymbol{M}}
$$

where $\gamma$ and $\boldsymbol{H}_{\text {eff }}$ denote the gyro-magnetic ratio and the effective magnetic field acting on $\boldsymbol{M}$, respectively. The second term on the right hand side of eq. (1) is called the Gilbert damping term, and the coefficient $\alpha$ is called the Gilbert damping constant, where the reversal time is proportional to $\alpha+1 / \alpha$ in bulk systems. In previous studies, $\alpha$ has been treated as a phenomenological parameter to discuss the magnetization processes of conventional objects. Since there is a rapidly growing interest in controlling the damping behavior, a study on the microscopic origin of $\alpha$ is required in the field of spintronics ${ }^{2}$ and for the developing new magnetic devices. Apart from works on Gilbert damping itself, there have been many theoretical works on the microscopic origin of the spin relaxation time. In 1954, Elliott ${ }^{3}$ ) suggested that if both the spin-orbit and electron-lattice interactions are taken into account, then the spin relaxation time is $1 / \tau_{S} \propto(g-2)^{2} / \tau_{R}$, where $\tau_{R}$ denotes an electron-lattice relaxation time. The effect of the spin-orbit interactions is revealed through the g-factor, which deviates from the value 2 in the presence of an orbital moment caused by the spin-orbit interactions. Spin-orbit interactions appear to be successful in explaining the temperature dependent line-width of the conduction electron resonance (CESR) spectra from the several non-magnetic metals observed by Beuneu et al. ${ }^{4)}$ Later, Fulde et al.5) and Singh $^{6)}$ gave a microscopic description of both the spin relaxation time and spin diffusion coefficient by considering the Coulomb interaction between the electrons and impurity scattering that accompanied the spin flip due to spin-orbit interactions. These theories are based on the concept that the spin damping is caused by the spin flip due to magnetic impurities (Khono, ${ }^{7)}$ Skadsem $^{8)}$ ) or spin-orbit interactions around the impurities (Yafet ${ }^{9)}$ ). However, Kambersky ${ }^{10)}$ proposed that the electronic structure changes depending on the moment direction, and thus, that the phase-lag of the electronic structure is revealed in the spin relaxation time. A similar mechanism was proposed by Korenman-Prange. ${ }^{11}$ Despite such differences, all these theories rely on the spin-orbit interaction in order to establish the transfer of spin angular momentum to an uncertain environment 
system.

In 2007, Kambersky ${ }^{12)}$ and Gilmore et al. ${ }^{13,14)}$ developed a theory based on the torque-correlation model to provide the quantitative aspect of $\alpha$ using first-principles calculations, which includes the spin-orbit interactions. In 2011, Ebert et al. ${ }^{15)}$ expressed $\alpha$ using the linear response theory based on the scattering theory developed by Brataas et al. ${ }^{16)}$ In addition, they quantitatively replicated the $\alpha$ of transition metal alloys by the first-principles approach using the Korringa-Kohn-Rostoker (KKR) method. The linear response theory enables us to apply the Green's function technique to calculate $\alpha$ for disordered alloys within the frame work of coherent potential approximation (CPA). As a result, the scattering effects due to the random arrangement of atoms are automatically taken into account. Starikov et al. ${ }^{17)}$ also performed the calculation of $\alpha$ for disordered transition metal alloys, based on the scattering theory. ${ }^{16)}$ Their expression of $\alpha$ is equivalent to those of Skadsem et al. ${ }^{8)}$ and Simanec et al. ${ }^{18)}$ in which $\alpha$ is proportional to the imaginary part of the spin susceptibility of electronic system. Furthermore, Kambersky ${ }^{12}$ ) showed that their expression is equivalent to the form given by the torque-correlation model.

In this article, we first review the basic concept of Gilbert damping by taking into account the theories mentioned above and theoretical framework describing the damping constant. Next, we present our recent results ${ }^{19)}$ for transition metals, including substitutional disordered alloys by using the CPA in the tight-binding linear muffin-tin orbital (TB-LMTO) method. For the calculation, we focus on the effects of the chemical degree of order on $\alpha$ for some transition metal alloys, which are important materials for spintronics devices. According to Kambersky, $\alpha$ behaves as $\alpha \propto \tau$ and $\alpha \propto 1 / \tau$ in the weak and strong scattering regions, respectively, where $\tau$ is the lifetime of the electrons due to electron-lattice interaction. In the present work, we aim to clarify how the scattering due to the random arrangement of atoms affects magnetization damping in acutual systems. Another concern of our work is to determine the dependence of $\alpha$ on the Fermi level in the rigid band scheme. We consider that this analysis will be useful because it is expected not only to yield the relationship between $\alpha$ and the electronic structure but also to help us to validate the results by comparing the spectra with those obtained by other methods or groups.

\section{Microscopic description of Gilbert damping}

\subsection{Physical picture of the magnetic damping}

Let us first look over the theoretical frame of the Gilbert damping from microscopic viewpoint. Suppose that the magnetization $\boldsymbol{M}_{e q}$ in the equilibrium state starts to rotate and then is changed into $\boldsymbol{M}(t)=\boldsymbol{M}_{e q}+\delta \boldsymbol{M}(t)$ by an applied magnetic field. $\delta \boldsymbol{M}(t)$ can be regarded as a magnon in a certain sense, and may create electron-hole pairs with opposite spins in the conduction electron system. The lifetime of the magnon becomes finite, when this process is accompanied by energy losses, which corresponds to the situation that the electron-hole pairs have dynamics. Note, however, that the uniform $(\boldsymbol{q}=0)$ dynamics of the electron-hole pairs does not take place in the low energy limit, because the angular momentum conservation prevent the uniform change of the total spins. If, on the other hand, magnetic impurities or spin-orbit interaction exist in the system, uniform spin dynamics may be realized through the spin flip.

The low energy electronic excitation accompanied by this spin flip process decreases number of magnons and then the magnetization turns towards to the direction of the applied field. Thus the Gilbert damping requires mechanism providing spin flip or mixing (hybridization) of the spin states, of which the magnetic impurities or spin-orbit interaction play the role. Figure 1 shows schematic diagram representing the process of annihilation of magnon followed by creating the electron-hole pairs. When this process occurs in the low energy limit under $\boldsymbol{q}=0$, magnon has a finite lifetime and its probability just corresponds to the Gilbert damping constant. In the following, we will make a microscopic description of the Gilbert damping constant through the derivation of the LLG equation in the quantum mechanical scheme.

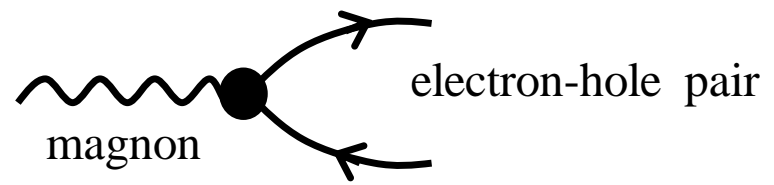

Fig. 1 Schematic diagram representing the magnon decay due to the creation of electron-hole pairs.

\subsection{Derivation of the LLG equation from the microscopic viewpoint}

From the above consideration, we understand that the magnetic relaxation of the Kittel mode (uniform magnetization precession) is caused by the electron spin excitation accompanied by the spin flip process through magnetic interactions. In 2003, Simanec et al. ${ }^{18)}$ described this process within the framework of the molecular field approximation. The magnetization inside the solid can be regarded as the order parameter with a classical vector once the system becomes a magnetically ordered state. When this system is broken and becomes a magnetic excited state, the deviation of the order parameter (magnetization) acts as a scattering center for the electrons, and a new interaction between the magnetization and the electrons as pseudo particles appear, even though the magnetization itself comprises electron spins. A convenient form of the Hamiltonian that can be used to describe this relaxation process is given by

$$
H(t)=H_{0}+H_{M-e}(t)+H_{Z}(t)
$$

where $H_{0}$ consists of the electron kinetic energy $H_{K}$, exchange splitting $H_{e x}=-(J / \gamma \hbar) \sum_{i} M_{z}\left(\sigma_{z}\right)_{i}$, and spin-orbit interaction $H_{S O}$. Here, $(J / \gamma \hbar)\left(M_{z}\right)_{i}$ represents the molecular (exchange) field with the order parameter $M_{z}$ pointing in the $\mathrm{z}$-direction, and $\sigma_{z}$ is the 
z-component of the Pauli matrix $\boldsymbol{\sigma} \cdot H_{Z}(t)$ represents the Zeemann energy $H_{Z}(t)=-\sum_{i} \boldsymbol{H}_{a p} \cdot \boldsymbol{M}_{i}(t)$ with applied field $\boldsymbol{H}_{a p}$ and the magnetization $\boldsymbol{M}_{i}(t)$ at the $i$ th site. We can express the time-dependent magnetization $\boldsymbol{M}_{i}(t)$ as $\boldsymbol{M}_{i}(t)=M_{z} \hat{z}+\delta \boldsymbol{M}_{i}(t)$ by using $M_{z}$ and its deviation $\delta \boldsymbol{M}_{i}(t)$. Here, we assume that the amplitude of the vector $\boldsymbol{M}_{i}(t)$ does not vary with time and that $\delta \boldsymbol{M}_{i}(t) \perp \boldsymbol{M}$. The second term $H_{M-e}(t)$ in eq. (2) is the interaction between the electron spins and the deviation of the order parameter $\delta \boldsymbol{M}_{i}(t)$ and can be expressed as

$$
H_{M-e}(t)=-(J / \gamma \hbar) \sum_{i} \delta \boldsymbol{M}_{i}(t) \cdot \boldsymbol{\sigma}_{i}
$$

In eq. (3), $J$ is the interaction strength, while $\sigma_{i}$ represents electron spin operator defined by $\boldsymbol{\sigma}_{i}=\sum_{\alpha, \beta} c_{i, \alpha}^{+} \boldsymbol{\sigma} c_{i, \beta}$, where $c_{i, \alpha}^{+} 、 c_{i, \alpha}$ are the electron creation and annihilation operators, respectively, of the $\alpha(=\uparrow, \downarrow)$ spin states at the $i$ th site.

Based on the Hamiltonian of eq.(2), the magnetization vector should obey the Bloch equation given by

$$
\dot{\boldsymbol{M}}_{i}(t)=\gamma \boldsymbol{M}_{i} \times\left(\boldsymbol{H}_{a p}+\frac{J}{\gamma \hbar}\left\langle\boldsymbol{\sigma}_{i}(t)\right\rangle\right)
$$

The expectation value $\left\langle\boldsymbol{\sigma}_{i}(t)\right\rangle$ is to be evaluated using the Hamiltonian of the electronic system $H_{0}+H_{M-e}(t)$ This can be done by using the linear response theory if one regards $\delta \boldsymbol{M}_{i}(t)$ as an external field. The resultant form of $\left\langle\boldsymbol{\sigma}_{i}(t)\right\rangle$ is

$$
\left\langle\boldsymbol{\sigma}_{i}(t)\right\rangle=\left\langle\boldsymbol{\sigma}_{i}\right\rangle_{0}+\sum_{j} \frac{J}{\gamma \hbar} \frac{i}{\hbar} \int_{0}^{\infty} d t^{\prime}\left\langle\left[\boldsymbol{\sigma}_{i}\left(t^{\prime}\right), \boldsymbol{\sigma}_{j}\right]\right\rangle_{0} \cdot \delta \boldsymbol{M}_{j}\left(t-t^{\prime}\right)
$$

where the expectation value $\langle\cdots\rangle_{0}$ is calculated using the Hamiltonian $H_{0}$, and where $\boldsymbol{\sigma}_{i}(t)=e^{i H_{0} t / \hbar} \boldsymbol{\sigma}_{i} e^{-i H_{0} t / \hbar}$ The first term $\left\langle\boldsymbol{\sigma}_{i}\right\rangle_{0}$ represents the expectation value in the absence of $\delta \boldsymbol{M}_{i}(t)$ and can be included in the term $\boldsymbol{H}_{a p}$ in eq. (4).

If one approximates $\delta \boldsymbol{M}_{j}\left(t-t^{\prime}\right) \cong \delta \boldsymbol{M}_{j}(t)-t^{\prime} \delta \dot{\boldsymbol{M}}_{j}(t)$ by assuming the Markovian process, eq. (5) can be written as

$$
\left\langle\boldsymbol{\sigma}_{i}(t)\right\rangle=\left\langle\boldsymbol{\sigma}_{i}\right\rangle_{0}+\frac{J}{\gamma \hbar} \sum_{j}\left\{\bar{\chi}_{i j}(0) \delta \boldsymbol{M}_{j}(t)+\left.i \frac{\partial}{\partial \omega} \bar{\chi}_{i j}(\omega)\right|_{\omega=0} \delta \dot{\boldsymbol{M}}_{j}(t)\right\}
$$

where $\bar{\chi}_{i j}(\omega)$ is the spin susceptibility tensor defined by

$$
\bar{\chi}_{i j}(\omega)=\frac{i}{\hbar} \int_{0}^{\infty} d t e^{i \omega t}\left\langle\left[\sigma_{i}(t), \sigma_{j}\right]\right\rangle_{0}
$$

Inserting eq. (6) into eq. (3) yields

$$
\begin{aligned}
& \dot{\boldsymbol{M}}_{i}(t)=\gamma \boldsymbol{M}_{i} \times \boldsymbol{H}_{i}^{e f f}+\boldsymbol{m}_{i} \times \sum_{j} \bar{\alpha}_{i j} \dot{\boldsymbol{M}}_{j} \\
& \boldsymbol{H}_{i}^{\text {eff }}=\boldsymbol{H}_{a p}+\frac{J}{\gamma \hbar}\left\{\left\langle\boldsymbol{\sigma}_{i}\right\rangle_{0}+\frac{J}{\gamma \hbar} \sum_{j} \bar{\chi}_{i j}(0) \delta \boldsymbol{M}_{j}\right\}
\end{aligned}
$$

$$
\bar{\alpha}_{i j}=-\left.i M \frac{J^{2}}{\gamma \hbar} \frac{\partial}{\partial \omega} \bar{\chi}_{i j}(\omega)\right|_{\omega=0}
$$

Here, we introduce the expression $\boldsymbol{m}_{i}=\boldsymbol{M}_{i}(t) / M$. Equation (8) is the general expression for the LLG equation where the damping term is non-local and the damping constant is given by a tensor. The tensor elements, $\alpha_{i j}^{x x}$ and $\alpha_{i j}^{x y}$ for example, are given by

$$
\begin{aligned}
& \alpha_{i j}^{x x}=-\left.M \frac{J^{2}}{\gamma \hbar} \frac{1}{2} \frac{\partial}{\partial \omega} \operatorname{Im} \chi_{i j}^{+-}(\omega)\right|_{\omega=0}=\alpha_{i j}^{y y} \\
& \alpha_{i j}^{x y}=-\left.M \frac{J^{2}}{\gamma \hbar} \frac{1}{2} \frac{\partial}{\partial \omega} \operatorname{Re} \chi_{i j}^{+-}(\omega)\right|_{\omega=0}=-\alpha_{i j}^{y x} \\
& \chi_{i j}^{+-}(\omega)=\frac{i}{\hbar} \int_{0}^{\infty} d t e^{i \omega t}\left\langle\left[\sigma_{i}^{+}(t), \sigma_{j}^{-}\right]\right\rangle_{0}
\end{aligned}
$$

Equations (10) and (11) reflect the physical scenario in that the magnetic damping constants are expressed by the spin susceptibility of the electronic system. Generally, $\operatorname{Re} \chi_{i j}^{+-}(\omega)$ is an even function of $\omega$, and hence, $\left.\frac{\partial}{\partial \omega} \operatorname{Re} \chi_{i j}^{+-}(\omega)\right|_{\omega=0}=0$. Given that system is isotropic, $\alpha$ at low temperatures is expressed as

$$
\begin{aligned}
\alpha_{i j} & =-\left.\frac{\gamma \hbar}{M} \frac{1}{2} \frac{\partial}{\partial \omega} \operatorname{Im} \frac{i}{\hbar} \int_{0}^{\infty} d t e^{i \omega t}\left\langle\left[J \frac{M}{\gamma \hbar} \sigma_{i}^{+}(t), J \frac{M}{\gamma \hbar} \sigma_{j}^{-}\right]\right\rangle_{0}\right|_{\omega=0} \\
& =-\frac{1}{4 \pi} \frac{J^{2} M}{2 \gamma \hbar} \operatorname{Tr}\left\{\sigma_{i}^{+}\left(G_{\mathrm{F}}^{+}-G_{\mathrm{F}}^{-}\right) \sigma_{j}^{-}\left(G_{\mathrm{F}}^{+}-G_{\mathrm{F}}^{-}\right)\right\}
\end{aligned}
$$

Here, we introduce the Green's function at the Fermi level $E_{\mathrm{F}}$, defined by

$$
G_{\mathrm{F}}^{ \pm} \equiv\left(E_{\mathrm{F}}-H_{0} \pm i \delta\right)^{-1}
$$

with infinitesimal positive value $\delta$. The benefit of using the Green's function technique is to help us to treat the lifetime of electrons by means of the perturbation expansion or the coherent potential approximation (CPA). In the latter case, the $\{\cdots\}$ part in eq. (12) should be replaced by $\langle\cdots\rangle_{\mathrm{av}}$ which denotes the configuration average of the impurities. Note, that eq. (12) is equivalent to the results obtained by Ebert et al. ${ }^{15)}$ who used the scattering theory developed by Brataas et al. ${ }^{16)}$

\subsection{Physical description of the Gilbert damping constant}

Now, let us examine the physical implication of eq. (12) and its relation to the results obtained previously by other researchers. If eq. (8) is transformed into a q-space, we obtain

$$
\begin{aligned}
& \dot{\boldsymbol{M}}_{q}(t)=\gamma \boldsymbol{M}_{q} \times \boldsymbol{H}_{-q}^{e f f}+\boldsymbol{m}_{-q} \times \alpha_{q} \dot{\boldsymbol{M}}_{q} \\
& \alpha_{q}=-\left.\frac{J^{2} M}{2 \gamma \hbar} \frac{\partial}{\partial \omega} \operatorname{Im} \frac{i}{\hbar} \int_{0}^{\infty} d t e^{i \omega t}\left\langle\left[\sigma_{q}^{+}(t), \sigma_{-q}^{-}\right]\right\rangle_{0}\right|_{\omega=0}
\end{aligned}
$$

where we introduce the expression

$$
\sigma_{q}^{+}=\sum_{i} \sigma_{i}^{+} e^{i q \boldsymbol{R}_{i}}=(2 / \sqrt{N}) \sum_{k} c_{\hat{k}}^{+} c_{k+q \downarrow} \cdot
$$

Using the identity $\sigma_{q}^{ \pm}=\mp(1 / 2)\left[\sigma_{q}^{ \pm}, \sigma_{z}\right]$, the matrix element of $(J / \gamma \hbar) M \sigma_{q}^{ \pm}$becomes 


$$
\begin{aligned}
& \left\langle n\left|(J / \gamma \hbar) M \sigma_{q}^{ \pm}\right| m\right\rangle=\mp\left\langle n\left|\left[s_{q}^{ \pm}, H_{e x}\right]\right| m\right\rangle \\
& \quad=\mp\left\langle n\left|\left[s_{q}^{ \pm},\left(H_{0}-H_{K}-H_{S O}\right)\right]\right| m\right\rangle \\
& \quad=\mp\left\langle n\left|\left[s_{q}^{ \pm}, H_{0}\right]\right| m\right\rangle \pm\left\langle n\left|\left[s_{q}^{ \pm}, H_{K}\right]\right| m\right\rangle \pm\left\langle n\left|\left[s_{q}^{ \pm}, H_{S O}\right]\right| m\right\rangle
\end{aligned}
$$

The first term is equal to $\mp\left(E_{m}-E_{n}\right)\left\langle n\left|s_{q}^{ \pm}\right| m\right\rangle$ where $|m\rangle$ and $|n\rangle$, and $E_{m}$ and $E_{n}$ are the eigenstates and eigenvalues of $H_{0}$, respectively. This term can be neglected since eq. (12) requires $\delta\left(\hbar \omega-E_{m}+E_{n}\right)$ and then $E_{m}-E_{n} \rightarrow 0$ in the low energy excitation limit $(\hbar \omega \approx 0)$. This is in the order of Zeemann energy and is much smaller than $(J / \gamma \hbar)$ and the spin-orbit coupling. The second term in eq. (16) is proportional to $q^{2}$ near $q \approx 0$, and thus, it also vanishes for the case of uniform dynamics $(q \rightarrow 0)$. For the non-uniform case, this $q^{2}$ term contributes to $\alpha_{q}$ only when impurity scatterings exist, reflecting the spin diffusion through the spin currents. Actually, in the presence of impurity scattering, eq. (15) can be calculated using the Green's function technique including vertex correction. In the absence of $H_{S O}$ (the last term in eq. (16)), the approximate result near $q \approx 0$ is given by

$$
\begin{aligned}
& \alpha_{q} \approx \frac{1}{\gamma \hbar S} \rho_{\mathrm{F}}\left(\frac{\Gamma_{m}}{\Delta_{m}^{2}+\Gamma_{m}^{2}}+C q^{2}\right) \\
& C=\frac{v_{F}^{2}}{12 \Delta_{m}^{2}} \pi \rho_{\mathrm{F}}\left\{n u^{2}+n_{m} u_{m}^{2}\left(5 S_{z}^{2}+6 S_{\perp}^{2}\right)\right\} \\
& \Delta_{m}=J M /(\gamma \hbar) \\
& \Gamma_{m}=2 \pi \rho_{\mathrm{F}} n_{m} u_{m}^{2}\left(S_{z}^{2}+S_{\perp}^{2}\right)
\end{aligned}
$$

where the quantities $\rho_{\mathrm{F}}$ and $v_{\mathrm{F}}$ are the density of states (DOS) and velocity, respectively, at the Fermi level. $u$ and $u_{\mathrm{m}}$ denote the potentials of the non-magnetic and magnetic impurities, respectively, and $n$ and $n_{m}$ are the concentrations of the impurities. $S_{z}$ and $S_{\perp}=\left(S_{x}^{2}+S_{y}^{2}\right)^{1 / 2} / 2$ represent the components of impurity spin $\boldsymbol{S}$. Equation (17) indicates that the coefficient of the $q^{2}$ term, $C$, consists of both non-magnetic and magnetic impurity potentials, which was predicted by Umetsu et al. ${ }^{20}$ ) Terkovnyak et al. ${ }^{21}$ ) have also shown this behavior by only taking into account the non-magnetic impurities. However, note that in the limit of $q=0, \alpha=\alpha_{q=0}$ becomes finite only when the magnetic impurity scatterings exist. The behavior expressed as $\alpha \propto \Gamma_{m} /\left(\Delta_{m}^{2}+\Gamma_{m}^{2}\right)$ is consistent with the results reported by Skadsem et al. ${ }^{8)}$ Khono et al. ${ }^{7)}$ also showed $\alpha \propto \Gamma_{m}$ which is valid for $\Gamma_{m}<<\Delta_{m}$. This can be reproduced also by replacing $(J / \gamma \hbar) M \sigma_{q=0}^{ \pm}$in eq. (15) by $\mp\left[s_{q=0}^{ \pm}, H_{i m}\right]$ with the magnetic impurity potentials $H_{i m}=-v_{S} \sum_{i} \boldsymbol{S}_{i} \cdot \boldsymbol{\sigma} \cdot$

Since we have found that the first and second terms vanish in the limit of $q \rightarrow 0$ (uniform precession) in eq. (16), we get

$$
(J / \gamma \hbar) M \sigma_{q=0}^{ \pm}= \pm\left[s_{q=0}^{ \pm}, H_{S O}\right]
$$

when $H_{i m}$ is sufficiently weak. Plugging eq. (18) into eq. (12) with $q=0$, we get

$$
\left.\alpha=-\frac{1}{4 \pi} \frac{\mu_{\mathrm{B}}}{N M^{z}} \operatorname{Tr} T^{+} \mathbb{G}_{\mathrm{F}}^{+}-G_{\mathrm{F}}^{-}\right)^{-}\left(G_{\mathrm{F}}^{+}-G_{\mathrm{F}}^{-}\right)
$$

where $T^{ \pm}=\left[s_{q=0}^{ \pm}, H_{S O}\right]=\xi\left(l_{z} s^{ \pm}-l^{ \pm} s_{z}\right)$. Using this formalism, we can understand the physical aspects of $\alpha$ when the spin-orbit interaction $H_{\mathrm{so}}=\xi \sum \boldsymbol{l}_{i} \cdot \boldsymbol{s}_{i}$ exists.

Let the eigenstate and corresponding eigenvalue of $H_{0}$ be $|\boldsymbol{k}, m\rangle$ and $E_{\boldsymbol{k}, m}$, respectively, in the absence of impurities. Then, the actual form of $\alpha$ is given by $\alpha=\frac{\pi \mu_{\mathrm{B}}}{N M^{z}} \sum_{\boldsymbol{k}} \sum_{n, m}\left|\left\langle\boldsymbol{k}, n\left|T^{-}\right| \boldsymbol{k}, m\right\rangle\right|^{2} \delta\left(E_{\mathrm{F}}-E_{\boldsymbol{k}, n}\right) \delta\left(E_{\mathrm{F}}-E_{\boldsymbol{k}, m}\right)$

where, $m, n$ are the band indexes of the electronic states. This yields the same equation for $\alpha$ as obtained by Kambersky ${ }^{12)}$ and Gilmore et al. ${ }^{13,14)}$ One can easily notice from eq. (20) that the two different bands $(m \neq n)$ can not contribute to $\alpha$, unless they degenerate exactly at the Fermi level, because their wave vectors $\boldsymbol{k}$ are restricted to be identical. Moreover, due to the effects of the spin-orbit interactions of the unperturbed states, the matrix elements within the same band $(m=n)$ can contribute to the value of $\alpha$. This leads to a divergence of $\alpha$ through the factor $\left[\delta\left(E_{\mathrm{F}}-E_{\mathbf{k}, m}\right)\right]^{2}$. An infinite $\alpha$ implies that the electronic system responds limitlessly in accordance with the dynamics of $\boldsymbol{M}_{q=0}$ without any resistance. Since the relaxation time $\tau_{m a g}$ of $\boldsymbol{M}_{q=0}$ governed by eq. (14) is proportional to $\alpha+1 / \alpha$, an infinite $\alpha$ would provide an infinite $\tau_{\text {mag }}$, which would freeze $\boldsymbol{M}_{q=0}$. In a certain sense, this behavior seems peculiar, because it is widely believed that a larger damping constant makes the lifetime shorter. The proportional relation of $\tau_{\text {mag }} \propto \alpha$ for $1 / \alpha \rightarrow 0$ can be easily understood if one regards the vector $\alpha \dot{\boldsymbol{M}}$ in the Gilbert damping term as a reaction field against the motion of $\boldsymbol{M}$ or the friction of $\boldsymbol{M}$ through the response of the electronic spin system. Thus, the quick (infinite) response of the electronic spin system slows the dynamics of $\boldsymbol{M}$.

In order to take into account the lifetime of electrons, Kambersky ${ }^{12}$ ) and Gilmore et al. replaced the factor $\delta\left(E_{\mathrm{F}}-E_{\mathbf{k}, m}\right) \delta\left(E_{\mathrm{F}}-E_{\mathbf{k}, n}\right)$ by the Lorentzian type function

$$
\delta\left(E_{\mathrm{F}}-E_{\mathbf{k}, m}\right) \delta\left(E_{\mathrm{F}}-E_{\mathbf{k}, n}\right) \rightarrow \frac{\Gamma / \pi}{\left(E_{\mathrm{F}}-E_{\mathbf{k}, m}\right)^{2}+\Gamma^{2}} \frac{\Gamma / \pi}{\left(E_{\mathrm{F}}-E_{\mathbf{k}, n}\right)^{2}+\Gamma^{2}}
$$

where $\Gamma$ represents the damping constant of the electrons due to electron- lattice interactions. In this case, the divergence of the $m=n$ term is suppressed, and instead the $m \neq n$ term can contribute to $\alpha$ with the help of a finite $\Gamma$. This is attributed to an overlap of different bands by the energy broadening due to the finite lifetime $1 / \tau=\Gamma / \hbar$ of the electronic states. In Fig. 2, we schematically show the $\Gamma$ dependence of $\alpha$, 
which includes the case wherein the spin-orbit interaction is absent. As mentioned above, there are two processes that contribute to $\alpha$; one is the intra-band transition through the spin-orbit interactions and the other is the inter-band transition that occurs with the help of the electron-lattice interactions. The former contribution exhibits proportionality to $\tau=\hbar / \Gamma$ which is similar to conductivity, while the latter is nearly linear in terms of $1 / \tau=\Gamma / \hbar$, which is similar to resistivity. If the energy splitting, defined by $\Delta$, of the two bands connected through the matrix element $\left\langle\boldsymbol{k}, n\left|T^{-}\right| \boldsymbol{k}, m\right\rangle$ is close to $\Gamma$, the latter behavior of $\alpha \propto \Gamma$ should be replaced by $\alpha \propto \Gamma /\left(\Delta^{2}+\Gamma^{2}\right)$. This stems from the same feature as indicated by the constant term $(q=0)$ in eq. (17).

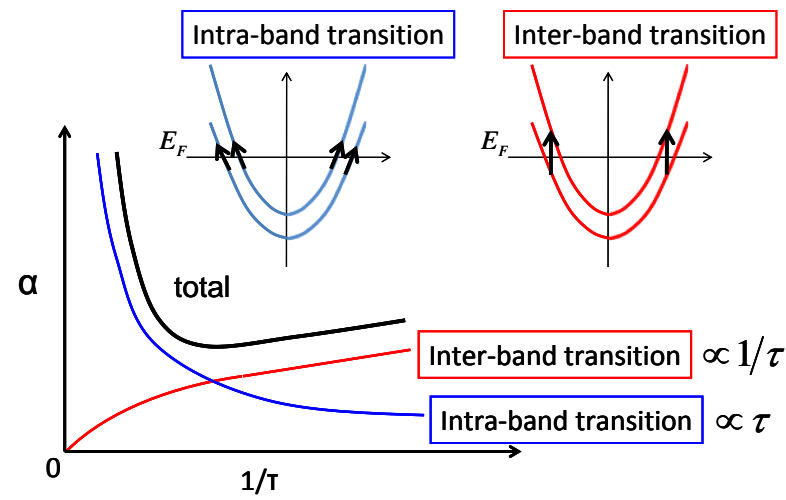

Fig. 2 Schematic diagram of $\alpha$ as a function of $1 / \tau=\Gamma / \hbar$ where $\tau$ is the electron lifetime. When the spin-orbit interaction is absent, only the inter-band transition can occur.

Based on eqs. (20) and (21) in the presence of impurities, Kambersky ${ }^{12}$ and Gilmore et al. ${ }^{14)}$ made a quantitative evaluation of $\alpha$ for $\mathrm{Fe}$, Co and Ni metals as a function of $1 / \tau=\Gamma / \hbar$, used as a phenomenological parameter in the first principles approach. Their results indicate that the $\alpha$ values of these metals have a minimum at around $1 / \tau \approx 10^{13}-10^{14} \mathrm{sec}^{-1}$. This can possibly explain the experimental results reported by Bhagat et al., ${ }^{22}$ ) according to which the damping constants of these metals exhibit a minimum at around $100 \mathrm{~K}\left(1 / \tau \approx 2 \times 10^{13} \mathrm{sec}^{-1}\right)$.

\section{Numerical calculation of the Gilbert damping constant $^{19)}$}

\subsection{Calculation method}

In the present section, we present some calculated results $^{19)}$ of Gilbert damping constants for transition metal systems using eq. (19). This form is useful because it is applicable to substitutional disordered alloys by using the CPA and electron lifetimes due to the random arrangement of atoms can be automatically introduced. For the electronic structure calculation, the TB-LMTO is used under the local spin density functional approximation (LSDA). When applying the CPA to eq. (19), we use the method developed by Butler ${ }^{23)}$ taking into account the vertex correction in the Kubo-Greenwood formula. The number of $\mathrm{k}$ points used in the calculation of $\alpha$ is approximately $5 \times 10^{5}$ in the full Brillouin zone. In principle, for the numerical calculation, the value of $\delta$ in eq. (13) should be selected as small as possible. However, when the system is close to the chemically ordered state where the scattering due to random arrangement is sufficiently weak, $\delta$ dominates the damping constant because it plays an inverse role of the lifetime of electrons in the completely ordered state. To estimate the appropriate value of $\delta$, we calculate the electrical resistivity of $\mathrm{Fe}$ and $\mathrm{Ni}$ as a function of $\delta$. Here we use the Kubo-Greenwood formula within the framework of TB-LMTO which was developed by Turek et al. ${ }^{24)}$

\subsection{Results and discussion}

Let us first examine the pure transition metal systems of $\mathrm{Fe}$ and $\mathrm{Ni}$. For this system, we need the finite $\delta$ to be the inverse of electron lifetime, $1 / \tau$. To determine the proper value of $\delta$, we first calculate the electrical resistivity of bcc-Fe and fcc-Ni using the Kubo-Greenwood formula. Figure 3 shows the calculated resistivity as a function of $1 / \tau(\delta)$ for $\mathrm{Fe}$ and $\mathrm{Ni}$. The allows indicate the measured values of the residual resistivity for each metal from which we can estimate $\delta$ of around $1-2 \mathrm{mRy}$ corresponding to $1 / \tau \approx 0.2-0.5 \times 10^{14} s^{-1}$. By varying $1 / \tau$ by an order of $10^{14} s^{-1}$, we plot $\alpha$ v.s. $1 / \tau$ for $\mathrm{Fe}$ and $\mathrm{Ni}$ (Fig. 4). One can see that $\alpha$ consists of two parts; one is $\alpha \propto \tau$ from the intra-band contribution and the another is $\alpha \propto 1 / \tau$ from the inter-band contribution. Around $1 / \tau \approx 0.2 \times 10^{14} s^{-1}$, the calculated $\alpha$ values for both $\mathrm{Fe}$ and $\mathrm{Ni}$ metals reasonably agree with the experimental values. From the results, we use $\delta=1$ mRy, because it provides the proper order for the residual resistivity (several $\mu \Omega \mathrm{cm}$ ) of the pure transition metal system.

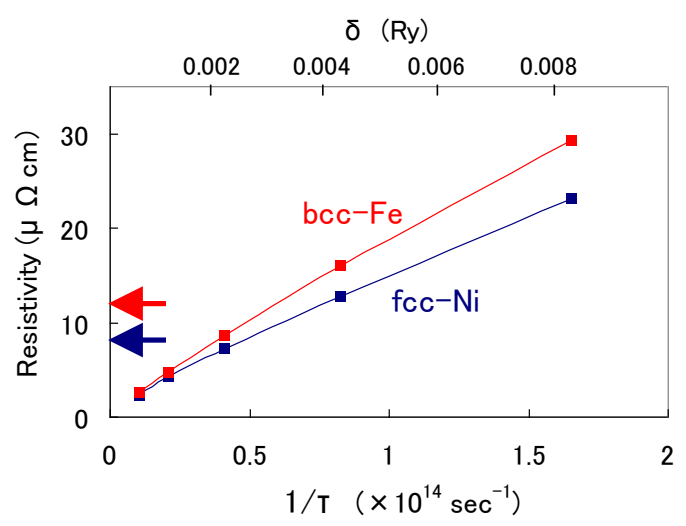

Fig. 3 Electrical resistivities of bcc-Fe and fcc-Ni as a function of electron scattering probability $1 / \tau(\delta)$, calculated using the Kubo-Greenwood formula under the TB-LMTO method. The arrows indicate the measured values of the residual resistivity for each metal,

Next, we evaluate the energy dependence of $\alpha$ (denoted by $\alpha(E)$ ) for $\mathrm{Fe}$ and $\mathrm{Ni}$ metals. $\alpha(E)$ corresponds to the variation in $\alpha$ when the Fermi level is shifted in the rigid band scheme, and it is useful 
to see the relationship between $\alpha$ and the electronic structure. In Fig. 5, we show the calculated results using $\delta=1 \mathrm{mRy}$. The origin of the horizontal axis corresponds to the real Fermi energy $E_{\mathrm{F}}$. The actual value of $\alpha$ is also given here. The spectral shapes of $\alpha$ for $\mathrm{Fe}$ and $\mathrm{Ni}$ are consistent with those proposed by Gilmore et al. ${ }^{14)}$, and this validates our calculation procedure. The actual $\alpha\left(E_{\mathrm{F}}\right)$ of each metal is approximately half of the experimental data. However, the trend that the $\alpha$ value of $\mathrm{Ni}$ is nearly ten times larger than that of $\mathrm{Fe}$ is consistent with the experimental results. This can be interpreted from the fact that the spin-orbit coupling of the $\mathrm{Ni}$ d-orbital $\xi_{d}$ is approximately two times larger than Fe. In addition, the Ni DOS has a sharp peak at the Fermi level. As shown by Gilmore et al., it seems that the spectral shape of $\alpha(E)$ in mono-atomic metals reflect the DOS to some extent.
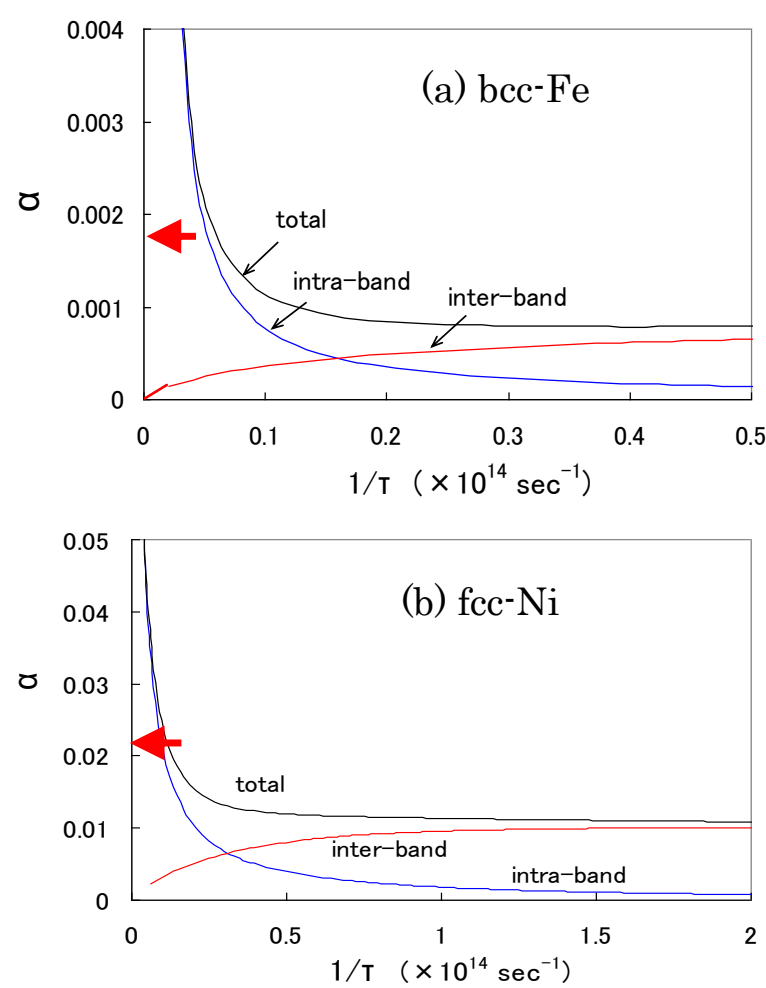

Fig. 4 Calculated Gilbert damping constants for (a) bcc-Fe and (b) fcc-Ni as a function of $1 / \tau$. The arrow indicates the measured value of each metal.

In the following text, we discuss alloy systems. Figure 6 shows the calculated result for $\alpha(E)$ of the disordered alloy, $\mathrm{Fe}_{0.2} \mathrm{Ni}_{0.8}$ (permalloy). Corresponding to the fact that the sharp peak in the DOS of pure $\mathrm{Ni}$ is diminished by the inclusion of $20 \% \mathrm{Fe}$, the peak of $\alpha\left(E_{\mathrm{F}}\right)$ in the case of the metal $\mathrm{Ni}$ disappears. Furthermore, the reduction of the averaged $\xi_{d}$ due to the inclusion of $\mathrm{Fe}$ is responsible for the decrease in $\alpha$. Consequently, $\alpha\left(E_{\mathrm{F}}\right)$ is reduced to approximately 0.0035 , which is nearly half that in the case of metal $\mathrm{Ni}$, and is close to the calculated result of Starikov et al. ${ }^{17)}$ Although this is approximately half of the value from the experimental data, ${ }^{25)}$ it is worth mentioning that the variation in $\alpha(E)$ in the entire energy range, shown in Fig. 6, is much larger than the discrepancy between $\alpha\left(E_{\mathrm{F}}\right)$ and the experimental value. This is also the case for Fe and Ni metals, as shown by Fig. 5 .
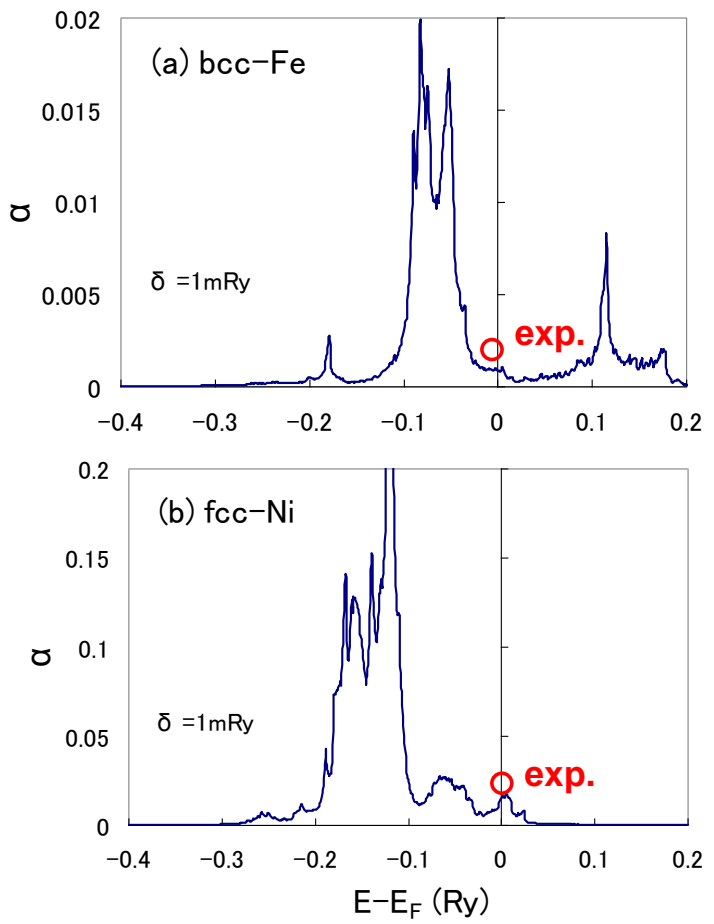

Fig. 5 Energy dependence of $\alpha(E$ ) for (a) $\mathrm{Fe}$ and (b) Ni with using $\delta=1 \mathrm{mRy}$. The actual $\alpha$ value is located at the Fermi level, $E_{F}$, the origin of the horizontal axis. The open circles represent the experimental data at room temperature. ${ }^{22)}$

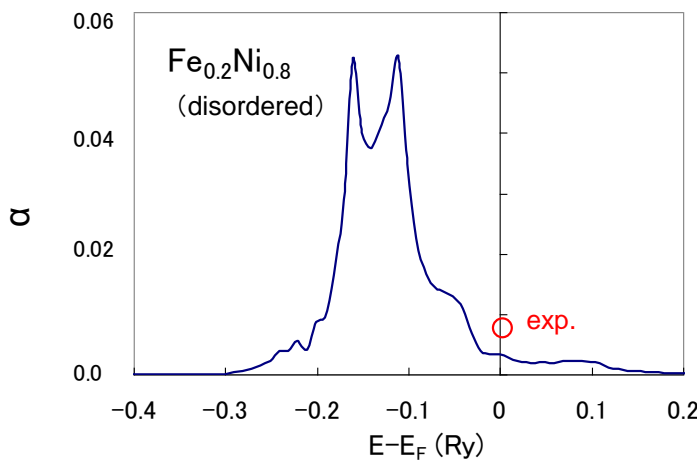

Fig. 6 The $\alpha(E)$ values of an $\mathrm{Fe}_{0.2} \mathrm{Ni}_{0.8}$ (permalloy) disordered alloy with $\delta=1 \mathrm{mRy}$. The open circle represents the experimental result. ${ }^{25)}$

In order to examine the magnitude dependence of $\xi_{d}$ on $\alpha$, we attempt addition of $\mathrm{Pt}$ atoms, whose $\xi_{d}$ is ten times larger than $\mathrm{Fe}$, and calculate $\alpha$ as a function of $\mathrm{X}$ in $\left(\mathrm{Fe}_{0.2} \mathrm{Ni}_{0.8}\right)_{1}$ - $\mathrm{Ptx}$. The results are shown in Fig. 7, along with the experimental data reported by Mizukami et al. ${ }^{26)}$ In both sets of data, $\alpha$ increased with $\mathrm{X}$, which can be understood from the matrix element average of $T^{ \pm}= \pm(i / \hbar)\left[S^{ \pm}, H_{S O}\right] \propto \xi_{d}$. 
We find from the LSDA calculation that the $\xi_{d}$ of Fe, $\mathrm{Ni}$ and $\mathrm{Pt}$ is approximately 50, 90, and $540 \mathrm{meV}$, respectively.

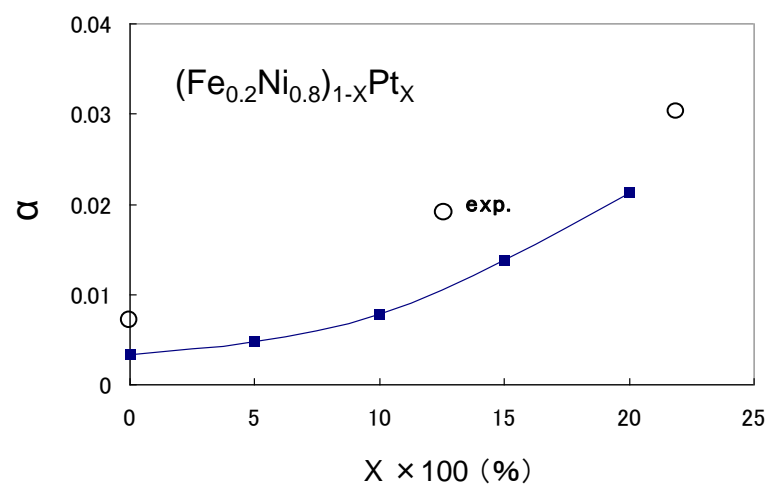

Fig. 7 Pt concentration dependence of $\alpha$ for $\left(\mathrm{Fe}_{0.2} \mathrm{Ni}_{0.8}\right)_{1}$ ${ }_{\mathrm{XPt}}$. The open circles represent the experimental result. ${ }^{26)}$

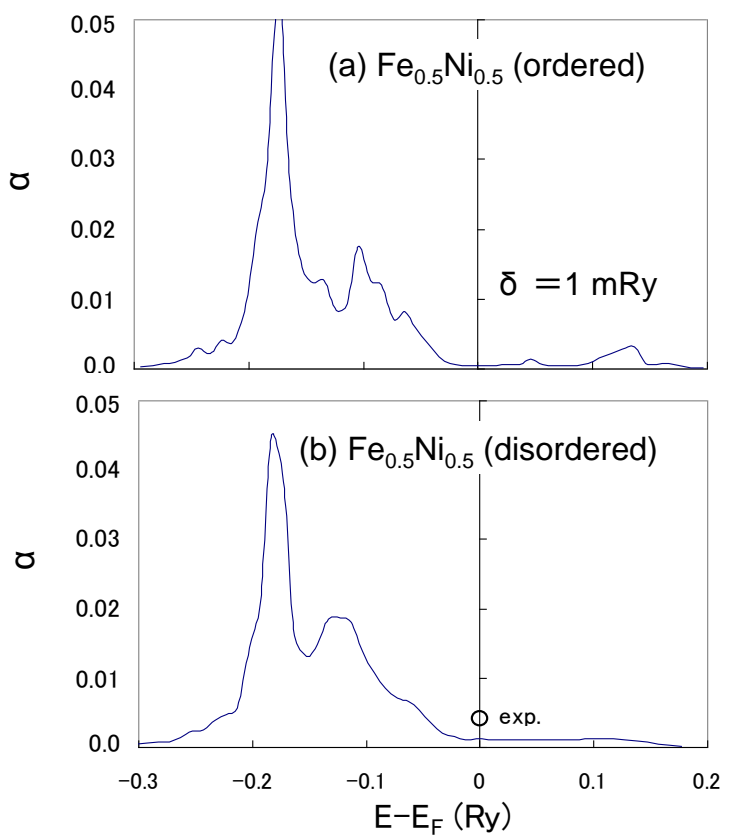

Fig. 8 The $\alpha(E)$ value (a) ordered and (b) disordered $\mathrm{Fe}_{0.5} \mathrm{Ni}_{0.5}$ alloys. The open circle represents the experimental result. $^{25)}$

To determine the relationship between $\alpha$ and the chemical degree of order, we focus on the equi-composition alloys, $\mathrm{Fe}_{0.5} \mathrm{Ni}_{0.5}$ and $\mathrm{Fe}_{0.5} \mathrm{Pt}_{0.5}$. $\mathrm{Fe}_{0.5} \mathrm{Ni}_{0.5}$ is a disordered alloy with an fcc structure in the stable state. However, the $\mathrm{L} 1_{0}$ type ordered alloy has recently been discovered in a meteorite ${ }^{27)}$ and its magnetic properties have attracted much interest. Although, $\mathrm{Fe}_{0.5} \mathrm{Pt}{ }_{0.5}$ alloys favor an $\mathrm{L}_{0}$ type ordered structure, in a thin film process, they often fall into a substitutional disordered state with an fcc structure and can be recovered by heat treatments. In Fig. 8, we show $\alpha(E)$ for ordered and disordered $\mathrm{Fe}_{0.5} \mathrm{Ni}_{0.5}$ alloys with $\delta=1 \mathrm{mRy}$. For disordered $\mathrm{Fe}_{0.5} \mathrm{Ni}_{0.5}$, we confirm that the result is not sensitive to $\delta$. This implies that the scattering strength due to the random arrangement is sufficiently large compared with $\delta$. It is found that $\alpha(E)$ for disordered $\mathrm{Fe}_{0.5} \mathrm{Ni}_{0.5}$ is further reduced when compared with $\mathrm{Fe}_{0.2} \mathrm{Ni}_{0.8}$ in the entire range of $E$, which may be attributed to the reduction of the average $\xi_{d}$ due to an increase in Fe concentration. In fact, the experimental $\alpha$ value of disordered $\mathrm{Fe}_{0.5} \mathrm{Ni}_{0.5}$ is approximately half that of $\mathrm{Fe}_{0.2} \mathrm{Ni}_{0.8}$. As also seen in the case of $\mathrm{Fe}_{0.2} \mathrm{Ni}_{0.8}, \alpha(E)$ in the range of -0.2 $\mathrm{Ry}<E-E_{\mathrm{F}}<^{-} 0.1 \mathrm{Ry}$ is much larger than $\alpha\left(E_{\mathrm{F}}\right)$, and the experimental values thus appear close to $\alpha\left(E_{\mathrm{F}}\right)$. The difference in the spectral shape of $\mathrm{Fe}_{0.2} \mathrm{Ni}_{0.8}$ may reflect the change in the DOS in which the Ni partial DOS is shifted toward the lower energy side due to the increase in $\mathrm{Fe}$ partial DOS on the higher energy side. Comparing the spectral shape of ordered and disordered structures, the disordered $\alpha(E)$ is somewhat spread out due to the finite lifetime of electrons. This may lead to an enhancement of the $\alpha\left(E_{\mathrm{F}}\right)$ value for the disordered phase, which corresponds to the $\alpha \propto 1 / \tau$ behavior shown in Fig. 2 .

To confirm the effects of the degree of order on $\alpha$, we introduce a parameter $\eta$ that varies from 0 to 0.5 for the form $\left(\mathrm{Fe}_{1-\eta} \mathrm{Ni}_{\eta}\right)_{0.5}\left(\mathrm{Fe}_{\eta} \mathrm{Ni}_{1-\eta}\right)_{0.5}$ in which $\eta=0$ indicates an $\mathrm{L} 10$ type ordered structure, and $\eta=0.5$ indicates a completely disordered fcc structure. In Fig. 9, we plot $\alpha$ for a $\left(\mathrm{Fe}_{1-\eta} \mathrm{Ni}_{\eta}\right)_{0.5}\left(\mathrm{Fe}_{\eta} \mathrm{Ni}_{1-\eta}\right)_{0.5}$ system for $\delta=0.3$ and $1 \mathrm{mRy}$ as a function of $\eta$, which can be regarded as the scattering strength due to the random arrangement of atoms. The results indicate that $\alpha$ is not sensitive to $\delta$ for $\eta>0.02$. As mentioned in the previous section, when the system approaches an ordered state $(\eta<0.02)$, the effect of $\delta$ becomes noticeable and exhibit a behavior expressed as $\alpha \propto 1 / \delta(\propto \tau)$. Therefore, as $\delta$ becomes smaller, $\alpha$ becomes larger when $\eta$ is close to 0 . For $\eta>0.02$, in contrast, $\alpha$ increases with an increasing $\eta$, although the variation is not very large and a small peak occurs at $\eta=0.4$. As shown in Fig. 2, the increasing behavior with $\eta$ can be understood from the inter-band transition with the help of the electron scattering due to the random arrangement. In addition, the peak at $\eta=0.4$ may reflect a resonance of the scattering probability with some inter-band transition energies across the Fermi level.

Finally, we discuss the case of $\mathrm{Fe}_{0.5} \mathrm{Pt}_{0.5}$. Figure 10 shows the $\alpha(E)$ values of a disordered structure. Due to the large $\xi_{d}$ of $\mathrm{Pt}$, the intensity is approximately ten times larger when compared with a $\mathrm{Fe}-\mathrm{Ni}$ system. In addition, the fact that the energy range of $\alpha(E)$ is wider than that of $\mathrm{Fe}-\mathrm{Ni}$ can be understood from the DOS of $\mathrm{Fe}_{0.5} \mathrm{Pt}_{0.5}$, where the $\mathrm{Pt}$ partial DOS spreads to a lower energy region compared with Ni. As in the Fe-Ni system, $\alpha\left(E_{\mathrm{F}}\right)$ remains at a much lower level compared to those around -0.4 Ry, which is below the Fermi level $E_{\mathrm{F}}$. We think this 


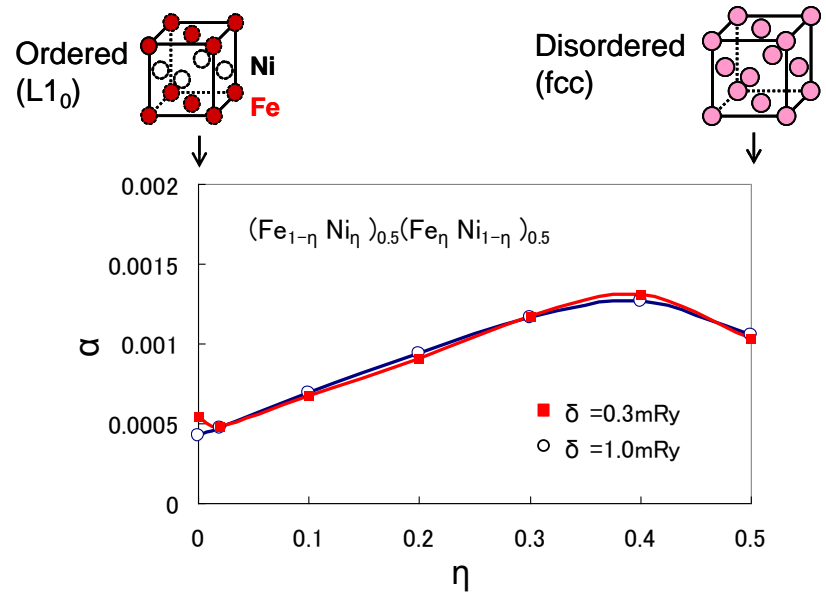

Fig. 9 The $\eta$ dependence of $\alpha$ in $\left(\mathrm{Fe}_{1-\eta} \mathrm{Ni}_{\eta}\right)_{0.5}\left(\mathrm{Fe}_{\eta} \mathrm{Ni}_{1-\eta}\right)_{0.5}$ structure, which represents the $\mathrm{L}_{0}$ ordered alloy when $\eta=0$, and the fcc disordered alloy for $\eta=0.5$.

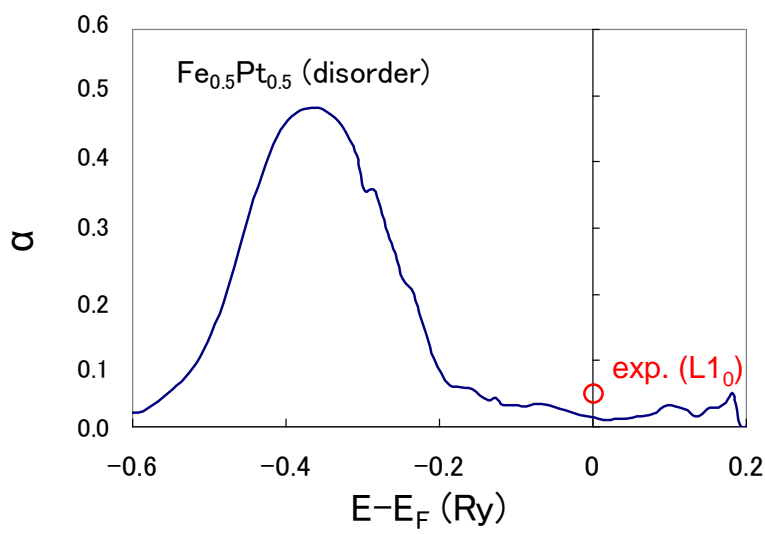

Fig. 10 The $\alpha(E)$ value of an $\mathrm{Fe}_{0.5} \mathrm{Pt}_{0.5}$ disordered alloy with an axial ratio of $c / a=1$. The open circle represents the experimental result ${ }^{26)}$ for an $\mathrm{L}_{0}$ ordered FePt alloy. ${ }^{28)}$

reflects the physical nature of the system, that is, the electronic structure is constructed such that it becomes difficult for the electron-hole pair excitation to occur at the real Fermi level. Figure 11 shows $\alpha$ as a function of $\eta$ for $\left(\mathrm{Fe}_{1-\eta} \mathrm{Pt}_{\eta}\right)_{0.5}\left(\mathrm{Fe}_{\eta} \mathrm{Pt}_{1-\eta}\right)_{0.5}$ with $\mathrm{c} / \mathrm{a}=1$ for $\delta=0.3$ and 1 mRy. Compared to the $\left(\mathrm{Fe}_{1-\eta} \mathrm{Ni}_{\eta}\right)_{0.5}\left(\mathrm{Fe}_{\eta} \mathrm{Ni}_{1-\eta}\right)_{0.5}$ system shown in Fig. 9, the dependence of $\alpha$ on $\delta$ is clearly seen in this figure in the region $\eta<0.02$. In this region, $\alpha$ grows rapidly with a decreasing $\eta$ for $\delta=0.3 \mathrm{mRy}$, whereas for $\delta=1$ mRy, the variation is not as large. This implies that the intra-band transition becomes stable process in this region, which is easily suppressed by $\delta$. When $\eta>0.02, \alpha$ increases with $\eta$ for both $\delta=0.3$ and 1 $\mathrm{mRy}$, in addition to the $\mathrm{Fe}-\mathrm{Ni}$ system. However, we note that the obtained values are much smaller than the measured value (0.06) even for the ordered FePt alloy $(\eta=0){ }^{28)}$ The smaller value of $\delta$ may provide larger $\alpha$ value near $\eta=0$. However, the parameter used here ( $\delta=0.3 \mathrm{mRy})$ is sufficiently small compared with the actual scattering rate in an actual system.

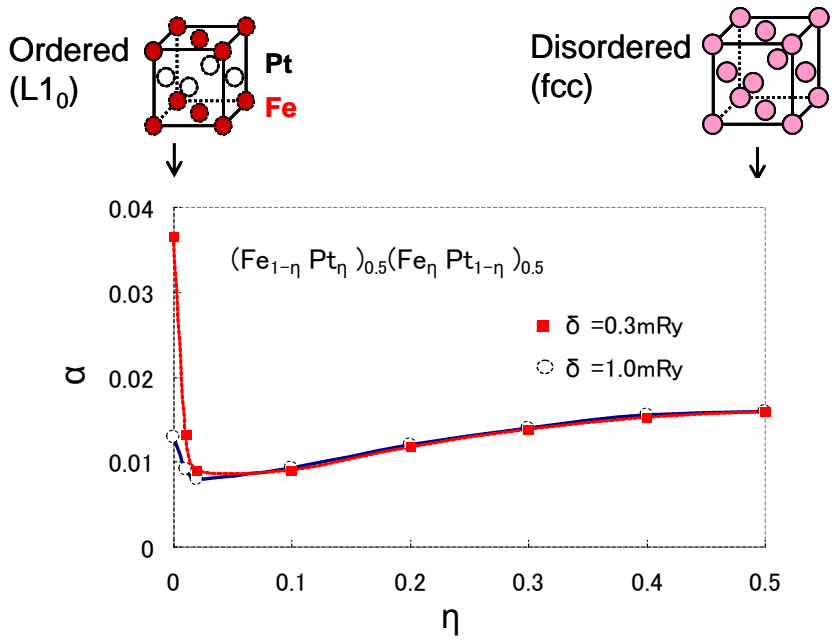

Fig. 11 The $\eta$ dependence of $\alpha$ in $\left(\mathrm{Fe}_{1-\eta} \mathrm{Pt}_{\eta}\right)_{0.5}\left(\mathrm{Fe}_{\eta} \mathrm{Pt}_{1-\eta}\right)_{0.5}$ structure with c/a $=1$ for $\delta=0.3$ and $\delta=1 \mathrm{mRy}$.

For $\mathrm{Fe}-\mathrm{Ni}$ and $\mathrm{Fe}-\mathrm{Pt}$ systems, the discrepancy in the value of $\alpha$ in the experimental data is considerably large, which indicates that the substitutional disordering effect is insufficient for the quantitative evaluation of $\alpha$. Therefore, further investigation is required, which can account for other effects such as thermal fluctuation of lattice and magnetic moments and, if necessary, the boundary condition of samples, such as their surfaces being adjacent to other systems.

\section{Summary}

In this article, we provide an overview of the basic concept of Gilbert damping including, which includes previous theories and theoretical framework describing the damping constant. It has been widely accepted that the damping constant can be expressed with the electron spin susceptibility using the molecular field Hamiltonian. We stress that for a uniform magnetic dynamics (Kittel mode), magnetic damping can occur only when magnetic impurities or spin-orbit interactions exist, while for a non-uniform case, damping can occur even if the impurities are non-magnetic. Next, we presented calculated results for the transition metal systems of $\mathrm{Fe}-\mathrm{Ni}$ and $\mathrm{Fe}-\mathrm{Pt}$ using the tight-binding linear muffin-tin orbital (TB-LMTO) method including spin-orbit interactions. In this calculation, the effects of the chemical degree of order on the $\alpha$ value of certain transition metal alloys, which are important materials in spintronics devices, are the main focus. We find that $\alpha$ decreases with an increasing chemical degree of order, whereas the variation is not very large. This can be interpreted as an increase related to the spin-flip channel due to magnetic scattering by the random arrangement of magnetic atoms. As expected, we confirm that in $(\mathrm{Fe}-\mathrm{Ni})_{1-\mathrm{xPtx}}$ and $\mathrm{FePt}$ systems, the elements having large spin-orbit coupling, such as $\mathrm{Pt}$ atoms, enhance the value of $\alpha$. We also examined the Fermi level dependence of $\alpha$ in the rigid band scheme. The 
results revealed that in both cases, $\alpha$ values at the real Fermi level remain at approximately half the value of the experimental result, whereas the variation in $\alpha(E)$ is much larger than these discrepancies.

Acknowledgments This work was partially supported by ASPIMATT program (JST) and JSPS KAKENHI Grant Number 25420686.

\section{References}

1) J. C. Slonczewski, J. Magn. Magn. Mater. 159, L1 (1996).

2) 1. Žutić, J. Fabian and S. D. Sarma, Rev. Mod. Phys. 76, 323 (2004).

3) R. J. Elliott, Phys. Rev. 96, 266 (1954).

4) F. Beuneu and P. Monod, Phys. Rev. B 18, 2422 (1978).

5) P. Fulde and A. Luther, Phys. Rev. 175, 337 (1968).

6) A. Singh and Z. Tesanovic, Phys. Rev. B 39, 7284 (1989).

7) H. Kohno, G. Tatara and J. Shibata, J. Phys. Soc. Jpn. 75, 113706 (2006).

8) H. J. Skadsem, Y. Tserkovnyak, A. Brataas, and G. E. W. Bauer, Phys. Rev. B 75, 094416 (2007).

9) Y. Yafet, Solid State Physics. 14, 1 (1963).

10) V. Kambersky, Can. J. Phys. 48, 2906 (1970).; J. Kunes and V. Kambersky, Phys. Rev. B 65, 212411-1 (2002).

11) V. Korenman and R. E. Prange, Phys. Rev. B 6, 2769 (1972).

12) V. Kambersky, Phys. Rev. B 76, 134416 (2007).

13) K. Gilmore, Y. U. Idzerda and M. D. Stiles, Phys. Rev. Lett. 99, 027204 (2007).

14) K. Gilmore, Y. U. Idzerda and M. D. Stiles, J. Appl.Phys. 103, 07 D303 (2008).
15) H. Ebert, S. Mankovsky, and D. Kodderitzsch, P. J. Kelly, Phys. Rev. Lett. 107, 066603 (2011).

16) A. Brataas, Y. Tserkovnyak, and G. E. Bauer, Phys. Rev. Lett. 101, 037207 (2008).

17) A. A. Starikov, P. J. Kelly, A. Brataas, Y. Tserkovnyak, G. E. W. Bauer, Phys. Rev. Lett. 105, 236601 (2010).

18) E. Simanec and B. Heinrich, Phys. Rev. B 67, 144418 (2003).

19) A. Sakuma, J. Phys. Soc. Jpn., 81, 084701 (2012).

20) N. Umetsu, D. Miura, and A. Sakuma, J. Phys. Soc. Jpn., 81, 114716 (2012).

21) Y. Tserkovnyak, E. M. Hankiewick, and G. Vignale, Phys. Rev. B 79, 094415 (2009).

22) S. M. Bhagat, P. Lubitz, Phys. Rev. B 10, 179 (1974).

23) W. H. Butler, Phys. Rev. B 31, 3260 (1985).

24) I. Turek, J. Kudrnovsky, V. Drchal, L. Szunyogh, and P. Weinberger, Phys. Rev. B 65, 125101 (2002).

25) M. Oogane, T. Wakitani, S. Yakata, R. Yilgin, Y. Ando, A. Sakuma, and T. Miyazaki, Jpn. J. Appl. Phys. 45, 3889 (2006).

26) S. Mizukami, T. Kubota, X. Zhang, H. Naganuma, M. Oogane, Y. Ando, and T. Miyazaki, Jpn. J. Appl. Phys. 50, 103003 (2011).

27) M. Kotsugi, C. Mitsumata, H. Maruyama, T. Wakita, T. Taniuchi, K. Ono, M. Suzuki, N. Kawamura, N. Ishimatsu, M. Oshima, Y. Watanabe, and M. Taniguchi, Appl. Phys. Express, 3, 013001 (2001).

28) S. Mizukami, S. Iihama, N. Inami, T. Hiratsuka, G. Kim, H. Nagahama, M. Oogane, Y Ando, Appl. Phys. Lett. 98, 052501 (2011).

Received Jul. 23, 2013; Accepted Aug. 19, 2013 